\title{
Tattoo preservation during surgical procedures
}

This article was published in the following Dove Press journal:

Open Access Surgery

17 February 2014

Number of times this article has been viewed

\section{Stefania Tenna \\ Pietro Francesco Delle \\ Femmine \\ Alfonso Luca Pendolino \\ Beniamino Brunetti \\ Paolo Persichetti \\ Plastic Surgery Unit, University Campus Bio-Medico of Rome, University of Rome, Rome, Italy}

Correspondence: Stefania Tenna Plastic Surgery Unit, University Campus Bio-Medico of Rome, University of Rome, via Alvaro del Portillo 200, 00 I I Rome, Italy Tel +39062 254I I220

Fax +390 62 254I 1936

Email s.tenna@unicampus.it
Abstract: In recent years, the number of people getting tattoos has continued to increase. Tattoos are much more than cultural fads and cosmetic complements, and nowadays often represent events that express the patient's personality without words. The presence of a tattoo in the surgical field may be a problem for both the patient and the surgeon. However, the relevant literature is mostly based on complications related to application of tattoos or methods used to remove them. To date, few reports have focused on the importance of preserving a tattoo during a surgical procedure, and no organized studies could be found. The aim of this paper is to provide an overview of the range of solutions that surgeons can use to preserve tattoos during surgery. A PubMed database search was done to assess other surgeons' experience. The terms "tattoo" in combination with "incision", "surgery", "surgical", or "operative" were used as key words. Following a review of the literature, photographs of patients presenting with a tattoo in the last 5 years at University Campus Bio-Medico of Rome were identified in order to determine the frequency of patients presenting with tattoos in our department. The patients were classified according to sex, age, type of surgery, number of tattoos, and tattoo location. Specific requests to preserve tattoos were recorded. Finally, an algorithm of treatment according to tattoo dimension and location is proposed. Knowledge of all the strategies available for saving tattoos is important for plastic and cosmetic surgeons. If a tattooed area needs to be operated on, surgeons should attempt, when possible, to avoid altering the tattoo in order to maximize the final cosmetic result.

Keywords: tattoo incision, body contouring, surgery, complications

\section{Introduction}

A tattoo is a design performed by introduction of exogenous pigments and/or dyes into the dermis. Derived from a Polynesian word ("tautau"), decorative tattooing has been found in most societies over a number of centuries. In recent years, the purpose of tattooing has varied from simple decoration, to a symbol of social rank, criminal and noncriminal group membership, in working categories such as coal mining, or a rite of passage in tribal communities. Over the last century, tattoos have become a means of self-expression, gaining popularity among adolescents, sometimes associated with high-risk behavior, and are common in the world of film and television celebrities and those in military service. ${ }^{1}$

In a recently published survey from the Walter Reed Army Medical Center, male soldiers reported receiving their first tattoo at an earlier age than female soldiers, and the most common tattoo described was a person's name. None regretted their tattoos, and had rarely been tattooed under the influence of alcohol or drugs. ${ }^{2}$ In fact, 20th 
century society's need for personal identity, uniqueness, and freedom of expression has resulted in a significant increase in the frequency of tattooing ${ }^{3}$ (Figure 1).

In 1936, it was estimated that 10 million Americans ( $6 \%$ of the population) had at least one tattoo, but tattoos have become increasingly common over the last decade. According to a survey in the fall of 2006 by the Pew Research Center, $36 \%$ of the American population aged $18-25$ years, $40 \%$ of those aged $26-40$ years, for a total of $14 \%$ of Americans, had at least one tattoo. The tattoo industry includes an estimated 20,000 parlors operating in the USA, with an annual revenue of $\$ 2.3$ billion. ${ }^{4}$ Interestingly, $32 \%$ of people with tattoos report that they are "ink-addicted" and $30 \%$ report that tattoos make them feel more physically appealing or rebellious. ${ }^{4}$

Nowadays, tattoos are considered a means by which to embellish the body and to effect cosmetic change, ${ }^{5}$ and are also becoming more frequent in medical practice, eg, to mark areas for radiotherapy, to reconstruct particular areas such as the areola or eyebrow, ${ }^{6}$ and in patients undergoing bariatric surgery because tattoos can hide scars and improve the body profile (Figure 2).

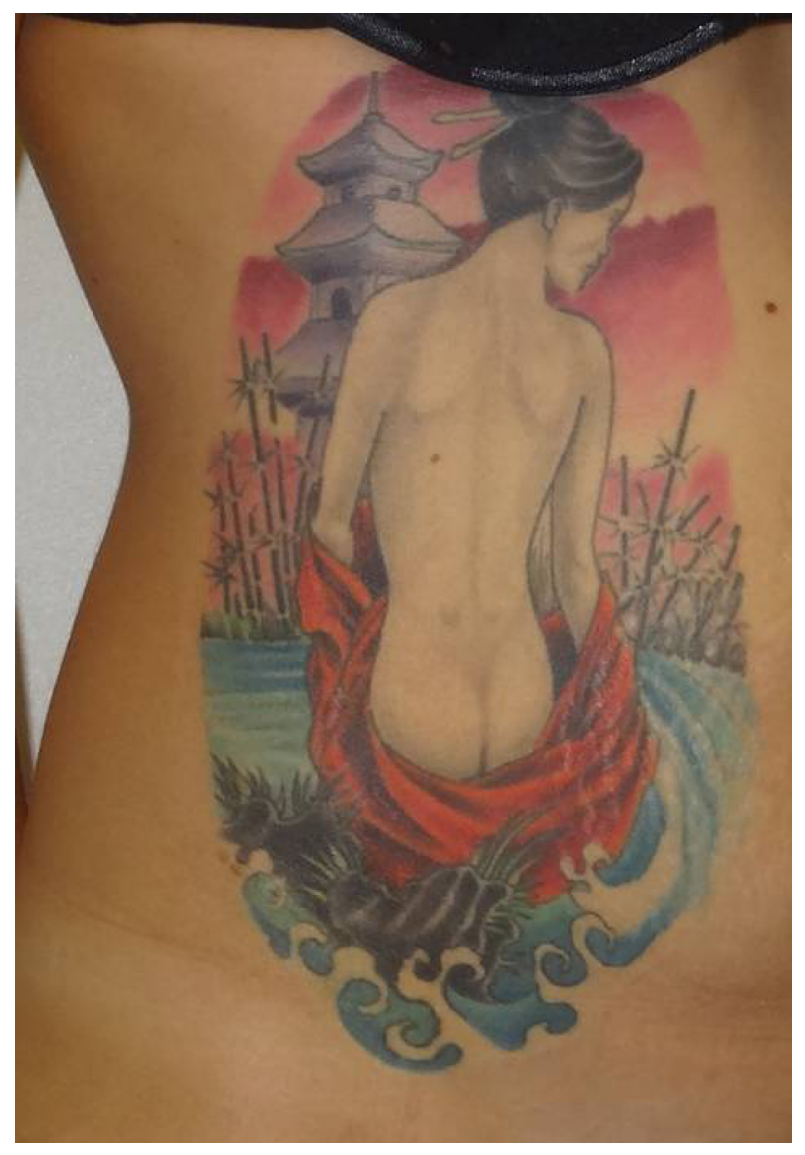

Figure I A 29-year-old female with a large colored tattoo involving the trunk.

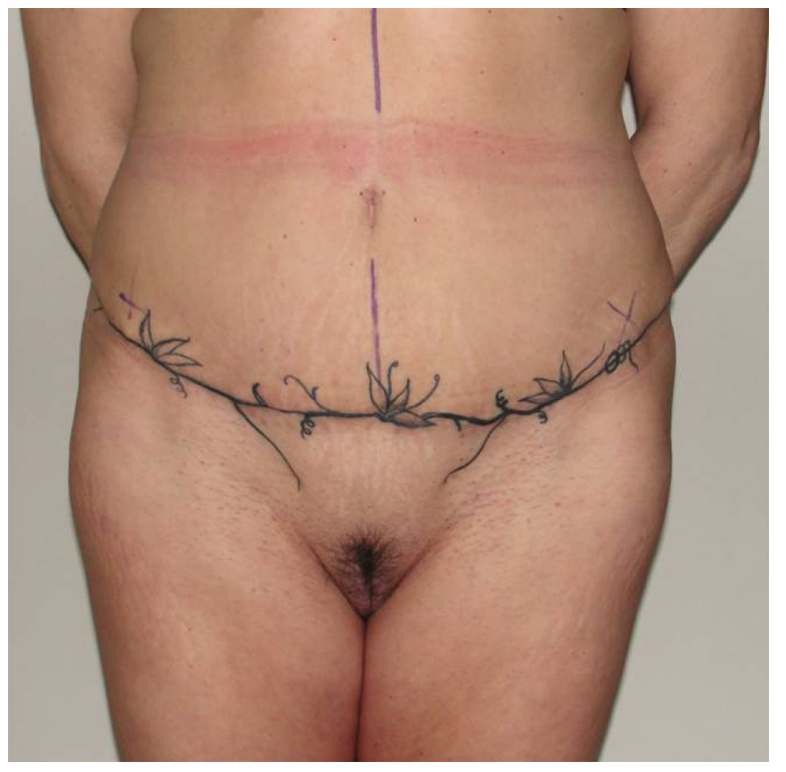

Figure 2 Clinical case of a 50-year-old female who had a tattoo made following an abdominoplasty to hide the scar.

As the popularity of tattooing continues to grow, some related issues arise. Complications may occur and adverse effects have been reported. Cases of infection, tattooassociated dermatoses, allergic reactions, and even development of malignant tumors have been described in the literature. ${ }^{5}$ Cases of allergic contact dermatitis as a result of temporary henna tattooing and cosmetic tattoos have also appeared in the recent literature.

Another issue is the increasing number of requests for tattoo removal, which has resulted in a need for researchers to explore safe, innovative, and efficacious removal methods. The options available for tattooing have been well described but must be defined for each patient. In terms of the way in which a tattoo is applied, different categories can be identified, eg, amateur, professional, cosmetic, and traumatic. ${ }^{1}$ Lasers continue to be a reliable and efficacious tool, but not every tattoo can be removed. Laser removal has variable results, depending on the type of tattoo and the colors used.

A further consideration is whether a tattoo can interfere with cosmetic surgery. Plastic surgeons are increasingly faced with this situation due to the increased prevalence of tattoos. Furthermore, a tattoo may be particularly important to the patient and may also be very useful in forensics for assisting in body identification if facial features or fingers have been damaged or altered. Moreover, aspects of a deceased person's history may be deduced from certain tattoos. ${ }^{6}$

For all these reasons, requests not to damage tattoos during surgery should be taken into consideration (Figure 3). Tattoos can interfere with surgical planning in many ways, 


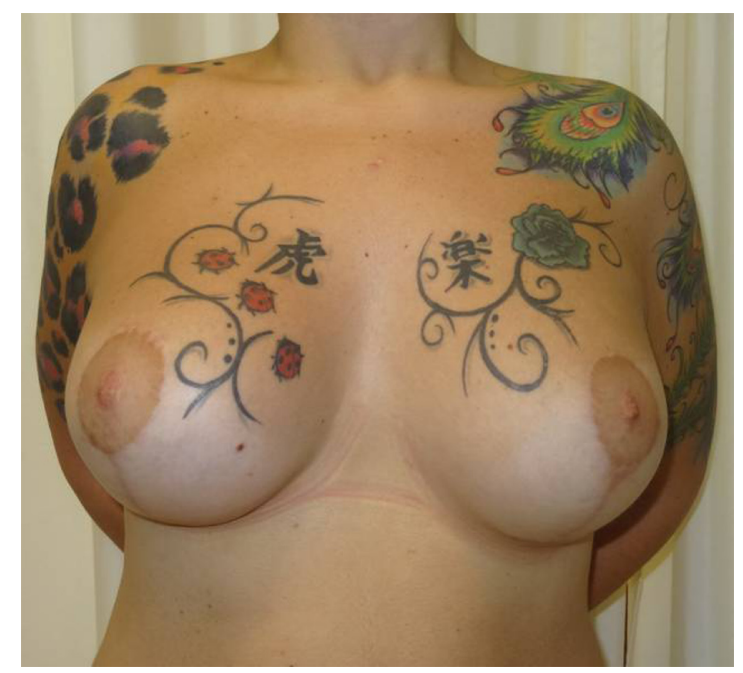

Figure 3 Large colored tattoos on shoulders, chest, and upper arm in a young woman who had undergone breast reduction and asked the surgeon not to alter the tattoos.

eg, they may be in the surgical field, located at incision sites, or involve different subunits, and may become distorted or partially altered in the event of undermining or resection.

In the case of surgery involving considerable resection (eg, mastopexy or abdominoplasty), an accurate operative strategy should be planned in order to minimize damage to tattoos and preserve their integrity. Thus, the surgeon must consider alternatives for every procedure, and several patterns or techniques have been described for a number of procedures, and offer the possibility of various resection options.

For example, there are many options available for reduction mammoplasty and mastopexy, depending on body habitus that may involve various types of skin resection, such as periareolar, vertical, "J", or "inverted T" techniques. Many other body-contouring procedures can also be performed by choosing the best pattern according to the position of the tattoo in order to allow the surgeon to preserve the tattoo without altering its appearance. However, the literature relates mostly to the complications associated with tattoo application, methods used for tattoo removal, and the potential complications after their removal. To date, only a few reports have focused on the importance of preserving tattoos during a surgical procedure, and no formal study could be found. The aim of this review is to provide an overview of the range of solutions available to the surgeon for preserving a tattoo during surgery.

\section{Materials and methods}

A PubMed database search was undertaken to assess the experience of other surgeons. The terms "tattoo" in combination with "incision", "surgery", "surgical", or "operative" were used as key words. Papers published between 1998 and April 2014 were considered for inclusion. References were screened for further relevant articles. Papers on tattoos related to cosmetic surgery were selected and critically evaluated.

After a review of the literature, photographs of patients presenting with a tattoo during the last 5 years at University Campus Bio-Medico of Rome were selected in order to assess the frequency of tattooing in patients coming to our department. Patients were classified according to sex, age, type of surgery, number of tattoos, and tattoo location. Specific requests to preserve tattoos were recorded. Preoperative and postoperative photographs were checked to assess tattoo integrity. A treatment algorithm according to tattoo dimension and location is presented.

\section{Results \\ Literature search}

A search of the literature revealed a total of 30 studies published between 1974 and 2014. Only five of these papers were strictly related to our topic. Of these, three were letters to the editor, one was an original article, and only one was a full review and published in 2006 . The patient population was not always reported, suggesting that this research related only to sporadic cases described in the literature. The range of techniques developed to preserve tattoos is still limited.

However, when considering the key word "tattoo" alone, more than 1,000 papers dealing with tattoo application, methods for tattoo removal, and complications following removal were found. The most significant papers found focused on complications related to tattooing.

Infections, chronic dermatoses, and hypersensitivity reactions to pigments (lichenoid or granulomatous) have been reported. ${ }^{7}$ Infectious complications occur usually within 4-22 days post-application and range from mild cellulitis and small pustules to abscesses. ${ }^{8,9}$ A case report describing a necrotizing cutaneous reaction after a permanent black tattoo was published in 2009 by Bhogal and Thomas. ${ }^{10}$

Local complications can usually be treated with specific topical medications. Topical steroids or antibiotics are often effective without surgery, although a surgical incision and/or drainage is sometimes required. In 2012, Fioramonti et al used a collagenase product to preserve a colorful tattoo following onset of ulcerative lesions. ${ }^{11}$ Use of collagenase increased wound debridement and facilitated the natural healing process. When topical therapy is not enough to solve the problem, an incision is often needed. 
Migration of tattoo pigment and/or silicone into the draining lymph nodes with development of lymphadenopathy has been reported. ${ }^{12}$ Many lesions can arise incidentally in tattooed skin or project onto it. Tumors are often coincidental lesions, ${ }^{13}$ and may be either benign or malignant, such as basal cell carcinoma, ${ }^{14}$ melanoma, ${ }^{15}$ keratoacanthoma, and other squamous cell variants. ${ }^{16,17}$

Various hypotheses have been put forward to explain why cancer could be more frequent in a tattooed area. The most worthwhile are those that recognize trauma and chronic inflammation to be common triggers in the development of tumors. ${ }^{18,19}$ Complications and benign or malignant tumors at the tattoo site often require further surgical intervention. Surgery may lead to total or partial excision of the tattoo, although, in other cases may require incision only. In the case of a partial excision, the surgeon must keep in mind all the possible options for preserving the tattoo, bearing in mind that any alteration could result in distortion of the intrinsic meaning of the tattoo. Touching up or complete removal of the tattoo is necessary in these cases. Reasons for tattoo removal include conditions of employment, stigma, a change in lifestyle or partner, incompatibility with current attitudes and values, and clothing issues. ${ }^{20-26}$ Data reported in the 2006 Pew Research Center survey indicate that $17 \%$ of Americans regret having been tattooed, and $11 \%$ of people with tattoos opt to undergo a removal procedure. ${ }^{4}$

There are presently several methods available for tattoo removal, including chemical (introduction of various caustic products into the dermis or application to the skin), mechanical (dermabrasion, salabrasion, cryosurgery, surgery), and thermal (Q-switched laser, infrared coagulation) approaches. ${ }^{20}$ Laser is the most popular method, but is not without complications. It is an expensive and time-consuming procedure, which often requires several sessions to achieve an acceptable result, and complete clearance of the tattooed area is rarely achieved. ${ }^{26-28}$
Professionally applied tattoos tend to penetrate the deeper layers of the skin at uniform levels. This uniformity allows surgeons to use techniques that remove broader areas of inked skin at the same depth. Homemade tattoos, which are often applied with an uneven hand, may be more difficult to remove. Moreover, several adverse effects have been reported, including purpura, crusting, blistering, infection, immediate darkening of the tattoo, deep collagen necrosis, and scarring ${ }^{29}$ (Figure 4).

In general, subjects who decide to undergo radical excision of a tattoo choose surgery. Surgery is a valid option, particularly for small tattoos located in areas of adequate skin laxity. ${ }^{30}$ However, the depth of the pigment in tattoos varies. Excision, as deeply as possible, with closure by suturing or skin grafting, or a combination of both, is probably the method most likely to achieve radical removal of all the pigment. ${ }^{31}$

\section{Patient population}

Between 2008 and 2013, almost 7,000 patients underwent surgical procedures in the plastic surgery unit at University Campus Bio-Medico of Rome, with 5,300 having outpatient surgery and 1,600 undergoing a major surgical procedure. Review of 2,500 photographs (preoperative and postoperative photographs are not taken routinely in outpatients) identified 175 patients with at least one tattoo $(7 \%)$.

Patients were classified according to sex, age, number of tattoos, tattoo location, tattoo size, color content of the tattoo, and type of surgery (Table 1). Tattoos were found more frequently in women, with a male to female ratio of 1:2. The age range of the patients was $30-50$ years, reflecting the emerging age pattern in tattooing (Figure 5). The upper arm was the most frequent site for tattoos, followed by the trunk, chest, abdomen, and back (Figures 6 and 7). Tattoos on the lower limbs were less common. Medium and large tattoos

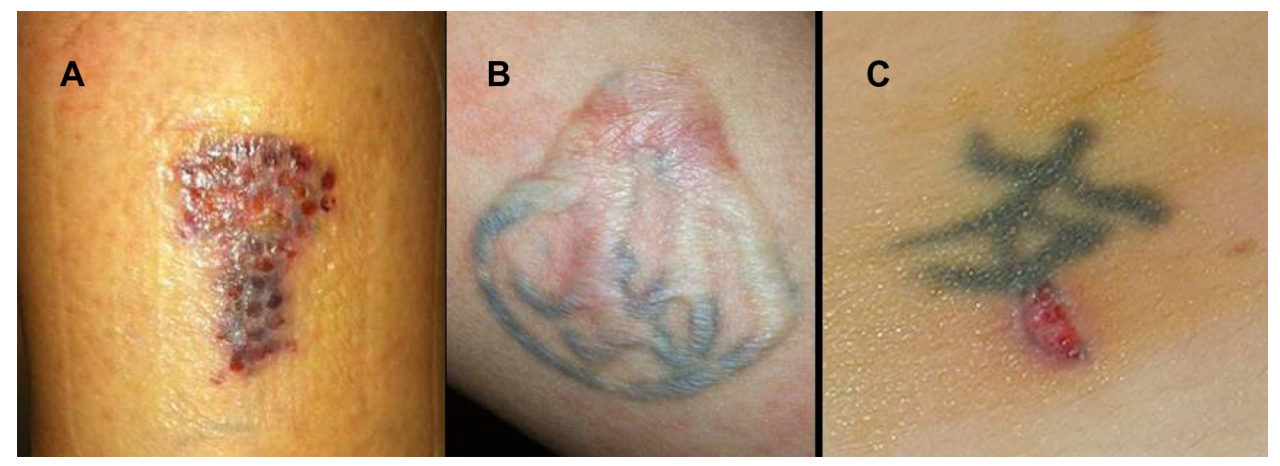

Figure 4 Complications related to removal of tattoos. (A) Blistering, (B) hypertrophic scarring, and (C) ulceration. 
Table I Patient population classified according to sex, age, number of tattoos, tattoo location, tattoo size, tattoo color, and type of surgery

\begin{tabular}{ll}
\hline Sex & \\
Male & $37.04 \%$ \\
Female & $62.96 \%$ \\
Age, years & \\
$\quad<40$ & $48.15 \%$ \\
$40-60$ & $48.15 \%$ \\
$>60$ & $3.70 \%$ \\
Number of tattoos & \\
I-2 & $83.33 \%$ \\
$3-10$ & $9.26 \%$ \\
$>$ I0 & $7.41 \%$ \\
Location of tattoo & \\
Neck & $4.62 \%$ \\
Upper arm & $43.08 \%$ \\
Inferior arm & $3.08 \%$ \\
Chest & $18.46 \%$ \\
Back & $7.69 \%$ \\
Abdomen & $16.92 \%$ \\
More than two regions & $6.15 \%$ \\
Size of tattoo & \\
Small & $31.48 \%$ \\
Medium & $35.19 \%$ \\
Large & $33.33 \%$ \\
Colored tattoo & \\
Yes & $31.48 \%$ \\
No & $68.52 \%$ \\
Type of surgery & \\
Abdoast surgery & $51.85 \%$ \\
Lesion removal & $20.37 \%$ \\
Other interventions & $9.26 \%$ \\
\hline & $18.52 \%$ \\
\hline
\end{tabular}

were more common than small tattoos, and black ink was preferred. Only 17 patients, amounting to $9 \%$ of the entire patient population, specifically requested tattoo preservation. Some patients required tattoo excision during surgery, but are not included in this review.
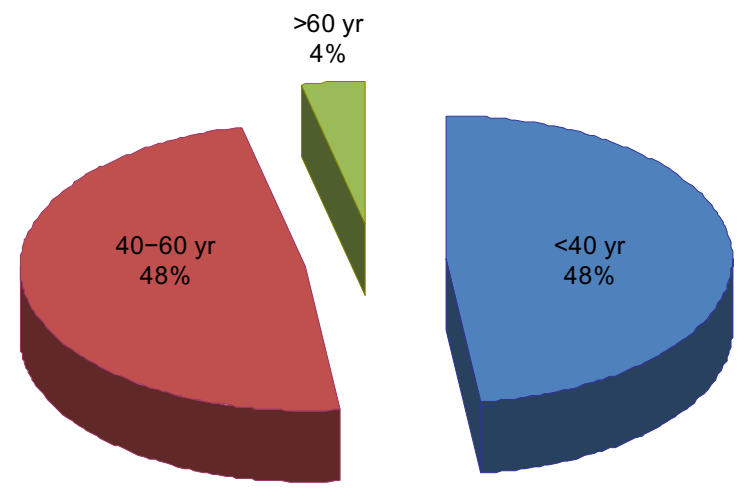

\section{Distribution of tattooed patients for age}

Figure 5 Distribution of patients according to age. Abbreviation: yr, year.

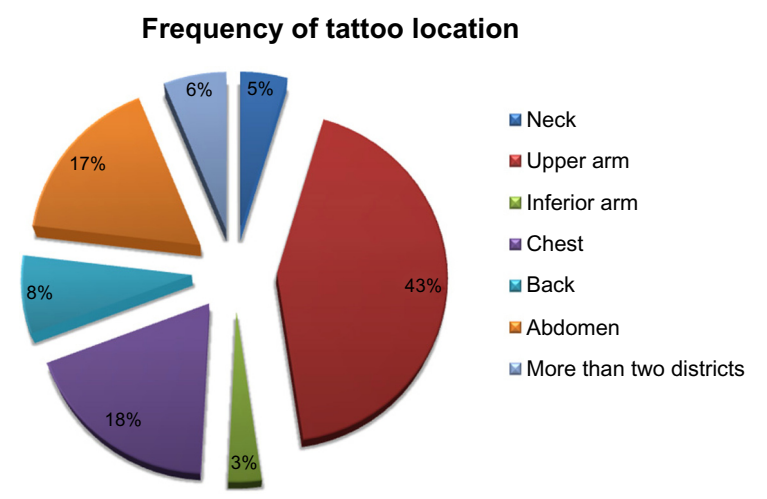

Figure 6 Distribution of tattoos according to anatomical location showing prevalence of the upper arm followed by the chest, abdomen, and back.

\section{Treatment algorithm}

Based on our experience in 175 patients, we have devised an algorithm for tattoo preservation according to tattoo dimensions and location (Figure 8). Type of tattoo, such as representative design, name of person, or tribal marks are considered secondarily, along with color, given that tattoos based on black ink are easier to remove by Q-switched laser. Three main groups of tattoos are considered, ie, small tattoos (regardless of color) located on the trunk, medium size tattoos on the extremities, and large colored tattoos involving different anatomical subunits.

For a small tattoo of limited dimensions (ie, a only few centimeters wide or long), there are no surgical limitations in terms of color or location (Figure 9). However, in the case

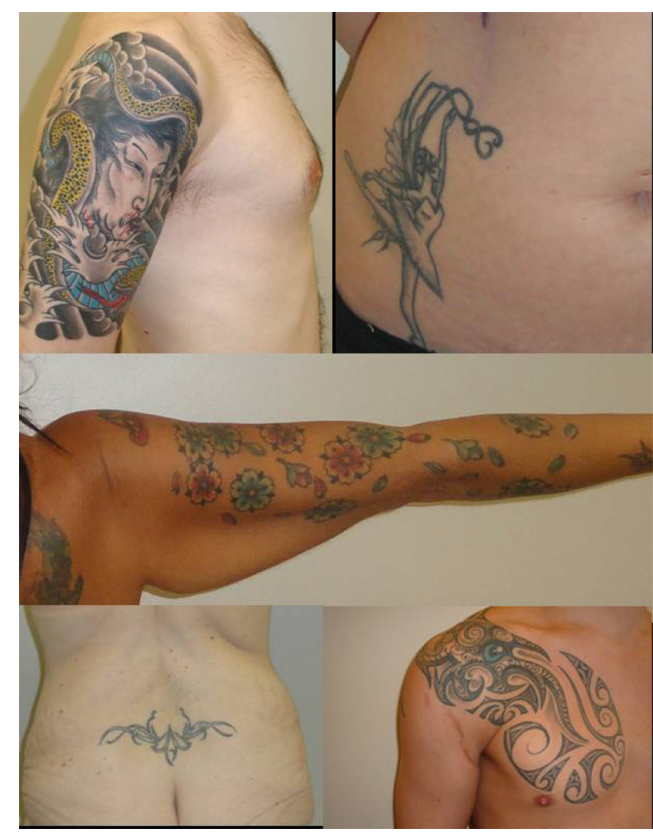

Figure 7 Variety of tattoos in different anatomical areas. 


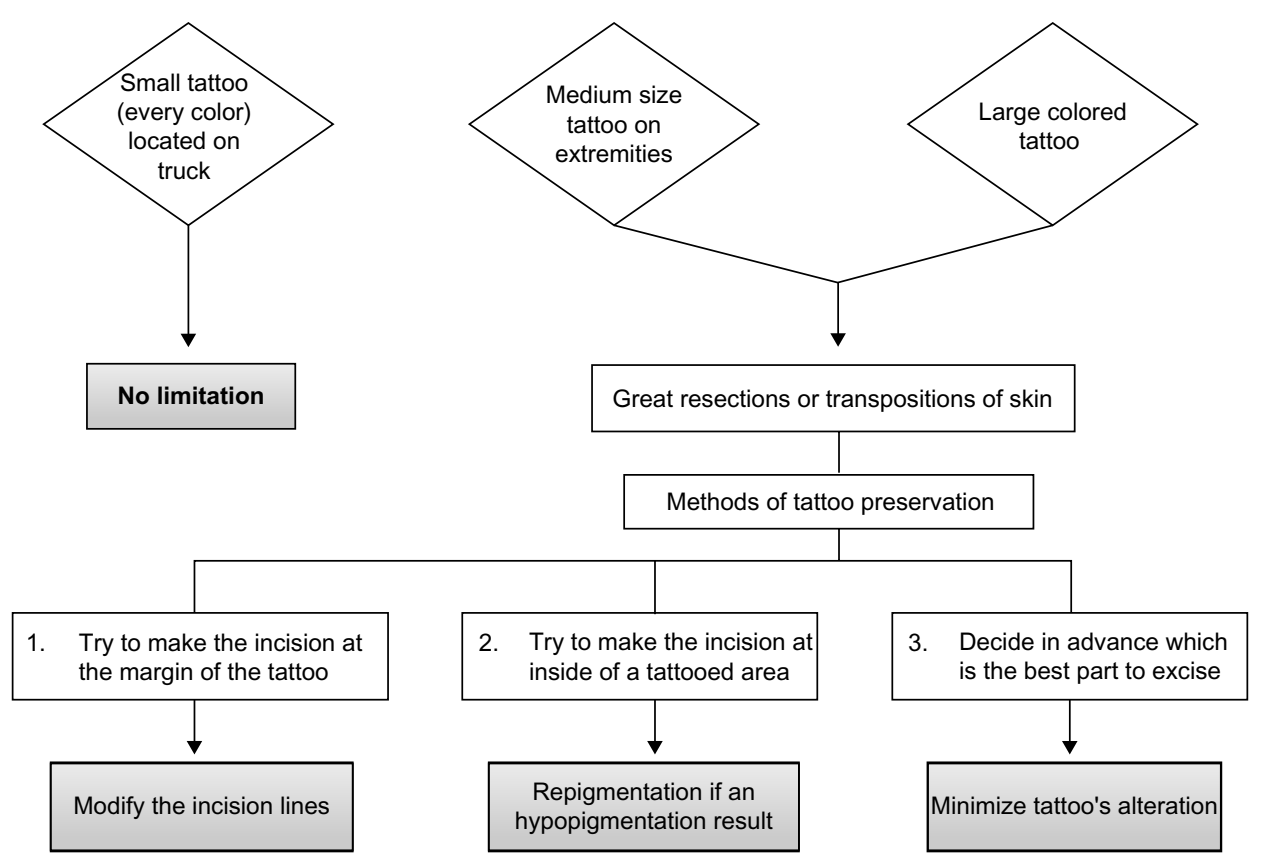

Figure 8 Algorithm of tattoo preservation according to tattoo dimension and location.

of a medium or large tattoo, surgeon must evaluate the best technique to adopt in consideration of the dimension and location of the tattoo (Figure 10).

Interventions that require large resection or transposition of skin, eg, abdominoplasty or flap harvesting, are more likely to involve a tattoo arising nearby. In these situations, alteration of the tattoo is often unavoidable, but accurate surgical planning may partially reduce this.

Methods to preserve a tattoo include three possible strategies: making the incision at the margin of the tattoo; making the incision inside the tattooed area; and a decision in advance about which is the best part to excise.

\section{Making the incision at the margin of the tattoo}

Incisions need not necessarily cause tattoo distortion. The surgeon can use a pre-existing tattoo in order to hide a

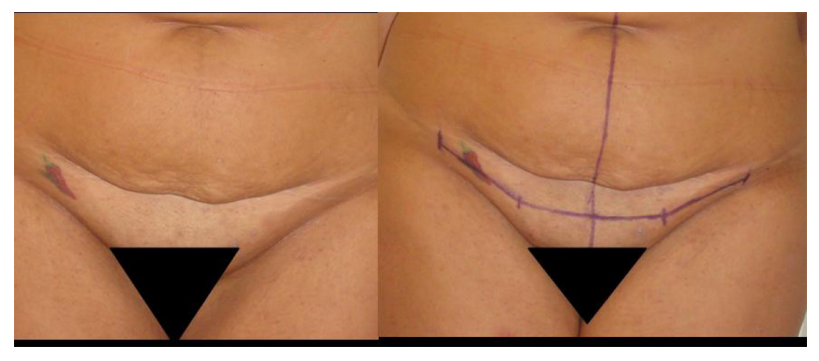

Figure 9 (A) Clinical case of a small tattoo on a young patient asking for abdominoplasty: (B) a modification of incision lines was planned in order to excise the tattoo. surgical incision. For example, the surgeon can attempt to make the incision at the margin of the tattoo in order to leave the scar along its contour. In this way, the tattoo can be viewed as an anatomical structure, in which the interface between the tattoo and normal skin is used to conceal the surgical incision and meet the need for patient satisfaction. ${ }^{32,33}$ These situations often require a modification of incision lines, as well as skin pattern removal, in order to prevent tattoo excision (Figure 11).

\section{Making the incision inside the tattooed area}

In the case of a tattoo with a large pigmented area, the surgeon can attempt to make the incision inside the tattooed area. If possible, an attempt should be made to make the incision at the interface between two colors. In this way, the excision will not significantly alter the features of the tattoo.

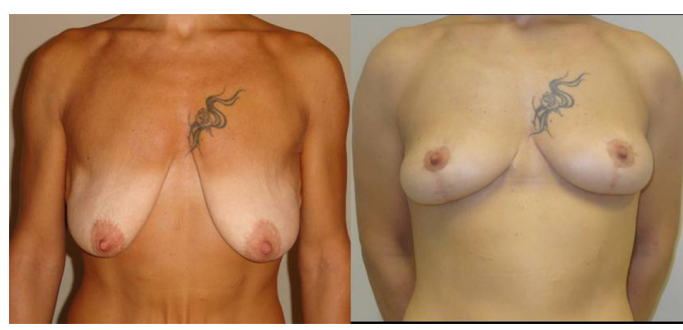

Figure 10 (A)Clinical case of a 40-year-old woman with a medium size tattoo on her chest undergoing mastopexy plus anterior intercostal artery perforator flap. (B) Postoperative result with the tatttoo that has not been altered. 


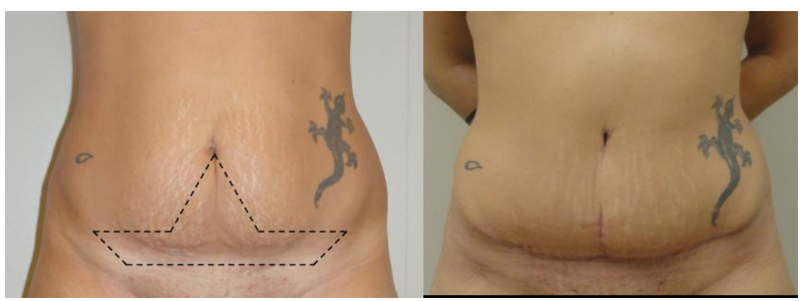

Figure I I (A) Clinical case of a 27-year-old woman undergoing abdominoplasty: (B) a modification of incision lines as well as skin pattern removal was done in order to prevent tattoo excision.

In addition, the hypopigmented scar can eventual be touched up and repigmented to hide the scar (Figure 12).

\section{Deciding on the best part to excise}

In cases where excision is unavoidable, the surgeon must carefully consider whether the tattoo should be excised totally or partially. If a partial removal is planned, the part of the tattoo that can be excised without severe alteration of the character of the tattoo must be evaluated in advance and discussed with the patient. In the case of a cutaneous lesion that could arise in the context of tattooed skin or for small tattoos involving areas that will be removed, the best choice

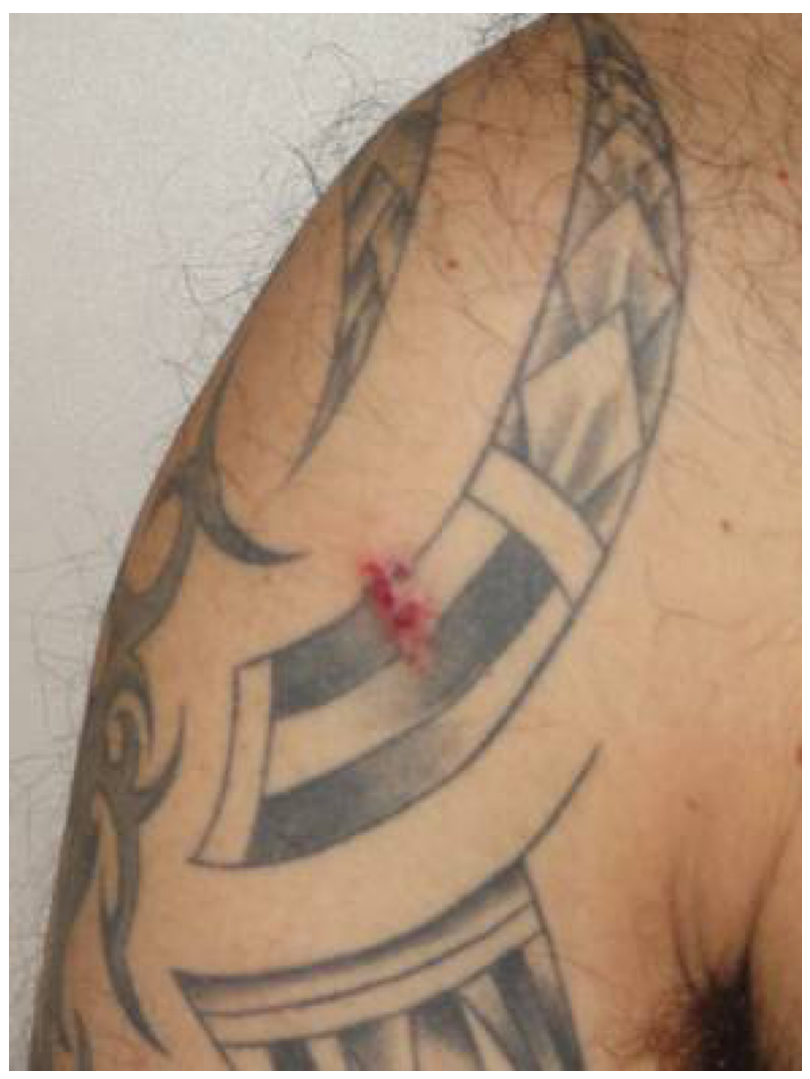

Figure 12 Treatment of a cutaneous cyst within a tattoo: the incision was carried out at the margin of the black fading. is complete removal. ${ }^{34}$ In the case of a subcutaneous lesion occurring under a tattoo, an excess of skin may sometimes result in skin expansion of a type that may negatively affect the tattoo, but total removal is preferable when possible.

When the tattoo is too large and cannot be saved, the injury should be as minimal as possible. Patients must be well informed regarding the pros and cons of intervention, especially if the procedure leads to damage of a tattoo with importance to the patient. Meticulous preoperative planning may help the surgeon to select which part of the tattoo can be excised without altering the cosmetic component and to make a correct preoperative marking (Figure 13). One option could be to excise the part of the tattoo that can be more easily retattooed following surgery in order to restore the part that is lacking. However, securing informed consent regarding the possibility of altering the appearance of the tattoo and the need to touch it up after surgery may avoid physicians becoming involved in medicolegal proceedings.

\section{Discussion}

Over recent years, the number of people deciding to become tattooed has steadily increased. Tattoos are much more than cultural fads or cosmetic additions, and nowadays often tell stories that express the patient's personality without words. The presence of a tattoo in the surgical field may be a problem for both the patient and the surgeon.

Following a review of the literature and personal experience, together with an increasing number of patients presenting with tattooed skin, we confirmed a proportional increase in numbers of lesions arising close to a tattoo and the presence of tattoos in the surgical field. Interestingly, it is not only young patients who present with tattoos; middleaged people also present with them, and often have multiple tattoos. We observed a higher frequency of tattoos in women

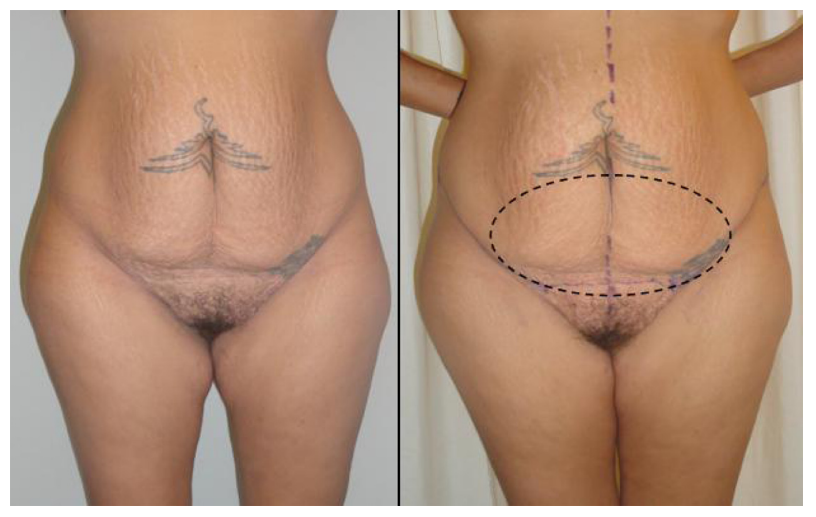

Figure 13 (A) Clinical case of a 37-year-old woman undergoing abdominoplasty. (B) In the area of excision some tattoos must be removed, then a touchup will be necessary. 
in our series, most of whom were presenting for abdominal procedures, but it must be borne in mind that female patients are more likely to request body-contouring interventions than men, and women account for the majority of our patient population. Among the types of surgery that need great resection or incision, which more often interfere with a tattoo, body-contouring procedures are the most frequently involved, especially abdominoplasty, where a large quantity of skin often needs to be excised. Consequently, long scars are more likely to alter a tattoo in a closer area. On the other hand, the concept of preserving a tattoo is still unusual, so patients rarely ask for them not to be altered. In 2011, the American Society of Dermatologic Surgery reported having performed almost 100,000 tattoo removal procedures, which is almost 20,000 more than in 2010. Nevertheless, complete tattoo removal is not always possible and, if laser treatment fails, surgical excision of the tattoo is the treatment of choice. In the case of large tattoos requiring serial excision, some degree of scarring or variation in skin color is a strong possibility. Essentially, plastic surgeons need to learn how to cope with tattoos.

Of course, in the emergency setting, saving a patient's life would take precedence over preservation of an attractive tattoo, but ruining a tattoo may not be acceptable when surgery is preplanned, even if the patient has felt uncomfortable about asking for preservation of a tattoo prior to surgery.

In our experience, we suggest that the reasons why the patient with a tattoo asks for surgery should be taken carefully into consideration. When surgery is a consequence of a complication related to a tattoo, a coincidental lesion arising in the area of the tattoo, or a patient's request for excision, no particular attention need be paid to the residual scar. However, if a skin resection that involves a tattooed area is required, the tattoo must be considered as an esthetic subunit. The patient could be so intimately attached to their tattoo as to interfere with surgical intervention, and distortion of a tattoo by surgical incision in such circumstances may be the basis for litigation. However, a patient with a tattoo does not easily agree to have surgery on it unless it is strictly necessary.

For all these reasons, we feel that plastic surgeons must take tattoos into account, particularly when approaching body-contouring procedures, giving precise information within the informed consent of all sequelae, and reminding these patients that a scar is not a tattoo, that surgery may alter the appearance of the tattoo, and that further camouflage interventions could be necessary. ${ }^{35,36}$

\section{Conclusion}

In the 21 st century, an era of esthetics and well-being, consideration of patient values and desires is of paramount importance for a plastic surgeon. A tattoo in the surgical field does not necessarily have to be sacrificed during surgery, and accurate planning of the procedure may help to preserve the integrity of a tattoo. Knowledge of all the strategies possible for saving a tattoo is important for plastic and cosmetic surgeons. If a tattooed area has to be operated on, surgeons should attempt, whenever possible, to save the tattoo to maximize the final cosmetic result.

\section{Author contributions}

ST, PFDF, and ALP contributed equally to the data collection, input, and analysis, and to the manuscript drafting and writing. $\mathrm{BB}$ and $\mathrm{PP}$ assisted with revising the paper, images and references. BB and PP were involved in the patients' operations. All authors read and approved the final manuscript.

\section{Disclosure}

The authors report no conflicts of the interest in this work.

\section{References}

1. Sweeney SM. Tattoos: a review of tattoo practices and potential treatment options for removal. Curr Opin Pediatr. 2006;18(4):391-395.

2. Lande RG, Bahroo BA, SoumoffA. United States military service members and their tattoos: a descriptive study. Mil Med. 2013;178(8): 921-925.

3. Tiggemann M, Golder F. Tattooing: an expression of uniqueness in the appearance domain. Body Image. 2006;3(4):309-315.

4. Pew Research Center. Vanishing Tattoo. 2012. Available from: http:// www.statisticbrain.com/tattoo-statistics/. Accessed January 18, 2014.

5. Körner R, Pföhler C, Vogt T, Müller CS. Histopathology of body art revisited - analysis and discussion of 19 cases. J Dtsch Dermatol Ges. 2013;11(11):1073-1080.

6. Byard RW. Tattoos: forensic considerations. Forensic Sci Med Pathol. 2013;9(4):534-542.

7. Sweeney SA, Hicks LD, Ranallo N, Snyder N 4th, Soldano AC. Perforating granulomatous dermatitis reaction to exogenous tattoo pigment: a case report and review of the literature. Am J Dermatopathol. 2013;35(7):754-756.

8. Miller LG, Perdreau-Remington F, Rieg G, et al. Necrotizing fasciitis caused by community-associated methicillin-resistant Staphylococcus aureus in Los Angeles. N Engl J Med. 2005;352(14):1445-1453.

9. Centers for Disease Control and Prevention. Methicillin-resistant Staphylococcus aureus skin infections among tattoo recipients - Ohio, Kentucky, and Vermont, 2004-2005. MMWR Morb Mortal Wkly Rep. 2006;55(24):677-679.

10. Bhogal RH, Thomas SS. Necrotizing black tattoo reaction: what's in a name? Am J Clin Dermatol. 2009;10(2):131-133.

11. Fioramonti P, Fino P, Ruggieri M, Scuderi N, Onesti MG. A successful collagenase and hyaluronic acid topical use combined with antibiotic therapy in the treatment of ulcerative lesions arising on tattoo. Case Rep Med. 2012;2012:253492.

12. Tehrani H, Lamberty BG. Axillary lymphadenopathy secondary to tattoo pigment and silicone migration. J Plast Reconstr Aesthet Surg. 2008;61(11):1381.

13. Kluger N, Plantier F, Moguelet P, Fraitag S. [Tattoos: natural history and histopathology of cutaneous reactions]. Ann Dermatol Venereol. 2011;138(2):146-154. French. 
14. Wiener DA, Scher RK. Basal cell carcinoma arising in a tattoo. Cutis. 1987;39(2):125-126.

15. Kirsch N. Malignant melanoma developing in a tattoo. Arch Dermatol. 1969;99(5):596-598.

16. Goldenberg G, Patel S, Patel MJ, Williford P, Sangueza O. Eruptive squamous cell carcinomas, keratoacanthoma type, arising in a multicolor tattoo. J Cutan Pathol. 2008;35(1):62-64.

17. Kluger N, Minier-Thoumin C, Plantier F. Keratoacanthoma occurring within the red dye of a tattoo. J Cutan Pathol. 2008;35(5):504-507.

18. Goldberg LH, Silapunt S, Beyrau KK, Peterson SR, Friedman PM, Alam M. Keratoacanthoma as a postoperative complication of skin cancer excision. J Am Acad Dermatol. 2004;50(5):753-758.

19. Janik JP, Bang RH. Traumatic keratoacanthoma arising in a 15 -year-old boy following a motor vehicle accident. Pediatr Dermatol. 2006;23(5): 448-450.

20. Burris K, Kim K. Tattoo removal. Clin Dermatol. 2007;25(4): 388-392.

21. Latreille J, Levy JL, Guinot C. Decorative tattoos and reasons for their removal: a prospective study in 151 adults living in South of France. J Eur Acad Dermatol Venereol. 2011;25(2):181-187.

22. Armstrong ML, Roberts AE, Koch JR, Saunders JC, Owen DC, Anderson RR. Motivation for contemporary tattoo removal: a shift in identity. Arch Dermatol. 2008;144(7):879-884.

23. Armstrong ML, Stuppy DJ, Gabriel DC, Anderson RR. Motivation for tattoo removal. Arch Dermatol. 1996;132(4):412-416.

24. Varma S, Lanigan SW. Motivation for tattoo removal. Arch Dermatol. 1996;132(12):1516.
25. Varma S, Lanigan SW. Reasons for requesting laser removal of unwanted tattoos. Br J Dermatol. 1999;140(3):483-485.

26. Wenzel S, Landthaler M, Baumler W. Recurring mistakes in tattoo removal. A case series. Dermatology. 2009;218(2):164-167.

27. Pfirrmann G, Karsai S, Roos S, Hammes S, Raulin C. Tattoo removal state of the art. J Dtsch Dermatol Ges. 2007;5(10):89-97.

28. Bernstein EF. Laser treatment of tattoos. Clin Dermatol. 2006;24(1): $43-55$.

29. Handley JM. Adverse events associated with nonablative cutaneous visible and infrared laser treatment. J Am Acad Dermatol. 2006;55(3):482-489.

30. Wollina U, Kostler E. Tattoos: surgical removal. Clin Dermatol. 2007; 25(4):393-397.

31. McDowell F. Editorial: Tattoo erasing. Plast Reconstr Surg. 1974; 53(5):580-581.

32. Lapid O. Use of an existing tattoo to conceal a surgical incision. Plast Reconstr Surg. 1998;102(7):2515-2516.

33. Tenna S, Delle Femmine PF, Filoni A, Cagli B, Brunetti B, Persichetti P. Management of tattoos in the operative field: how to save a gecko. Ann Plast Surg. 2012;69(1):112.

34. Inchingolo F, Tatullo M, Abenavoli FM, et al. Surgical removal of lipoma from an area with tattooed skin. Int J Med Sci. 2010;7(6):395-397.

35. Spyropoulou GA, Fatah F. Decorative tattooing for scar camouflage: patient innovation. J Plast Reconstr Aesthet Surg. 2009;62(10): e353-e355.

36. Kim EK, Chang TJ, Hong JP, Koh KS. Use of tattooing to camouflage various scars. Aesthetic Plast Surg. 2011;35(3):392-395.
Open Access Surgery

\section{Publish your work in this journal}

Open Access Surgery is an international, peer-reviewed, open access journal that focuses on all aspects of surgical procedures and interventions. Patient care around the peri-operative period and patient outcomes post surgery are key topics. All grades of surgery from minor cosmetic interventions to major surgical procedures are covered. Novel techniques

\section{Dovepress}

and the utilization of new instruments and materials, including implants and prostheses that optimize outcomes constitute major areas of interest. The manuscript management system is completely online and includes a very quick and fair peer-review system. Visit http://www.dovepress.com/ testimonials.php to read real quotes from published authors. 